\title{
ASYMPTOTIC CONSTANCY OF SOLUTIONS OF SYSTEMS OF DIFFERENCE EQUATIONS
}

\section{RIGOBERTO MEDINA}

Departamento de Ciencias

Universidad de Los Lagos

Casilla 933, Osorono, CHILE

\section{MANUEL PINTO}

Departamento de Matemáticas, Facultad de Ciencias Universidad de Chile, Casilla 653

Santiago, CHILE

(Received September 9, 1993 and in revised form October 31, 1994)

\begin{abstract}
Assuming only conditional summability we study the convergence of the solutions of difference systems
\end{abstract}

KEY WORDS AND PHRASES: Difference Systems, Weighted Norms, Convergent Solutions 1991 AMS SUBJECT CLASSIFICATION CODES: 34K20

\section{INTRODUCTION}

In [12], we have studied the asymptotic equilibrium of a general nonlinear difference equation

$$
\triangle x_{n}=f\left(n, x_{n}\right), \quad n \in N .
$$

After, we have studied the existence of convergent solutions of nonlinear systems whose linear part has a dichotomy See $[13,14,16-18]$ These results are obtained under absolutely summable conditions Motivated with the work of Trench [7] on differential equations we show that solutions of a system (l 1 ), approach constant vectors as $n \rightarrow \infty$, under assumptions which permit some or all of sum smallness conditions of $f$ to be stated in terms of conditional - rather than absolute convergence Through the paper the conditional convergence will be simply called convergence while the absolute convergence will be explicitly mentioned

This kind of problem for ordinary differential equations has been widely investigated by many authors, for example see $[1,4,6]$

It seems to us that very little is known about the convergence of the solutions of finite difference equations (see [15]) The only results that we know concerning this problem for second order difference equations are given by Drozdowicz and Popenda [2], Catillo and Pinto [3], Szmanda [5], Handerson and Peterson [8], Szafranski and Szmanda [9] and Medina and Pinto [10,11]

\section{PRELIMINARIES}

Consider the difference system (1.1), where $N=\left\{n_{0}, n_{0}+1, \cdots\right\}, n_{0}$ is a given non-negative integer, $x$ is an m-dimensional vector, $f: N \times \mathbf{R}^{m} \rightarrow \mathbf{R}^{m}$ is a function and $\mathbf{R}^{m}$ denote the mdimensional real Euclidean space, $\triangle$ is the difference operator, i e $\triangle x_{n}=x_{n+1}-x_{n}$ Throughout this paper the norm $|\cdot|$ of a vector or matrix is the sum of the absolute values of its elements

By a solution of Eq (1 1$)$ we mean any function $x$ defined on $N$, which fulfills Eq (1 1$)$ for all sufficiently large $n$ Note that the above definition of the solution is different from this where $x$ fulfills Eq (1.1) for all $n \in N$

Throughout this paper, we will suppose the following

ASSUMPTION. The $m \times m$ matrix function $V$ is non singular on $N$ and

Notice that (2 1) implies

$$
\sum_{j=n}^{\infty}\left|V(n) \triangle\left(V^{-1}(j)\right)\right| \leq K<\infty, \quad n \geq n_{0} .
$$


and so

$$
\sum_{\jmath=n_{0}}^{\infty}\left|\Delta\left(V^{1}(\jmath)\right)\right|<\infty
$$

$$
\lim _{n \rightarrow \infty} V^{-1}(n) \text { exists (finite) }
$$

LEMMA 1. If $q$ is an m-vector function on $N$ and $\sum_{j=n_{0}}^{\infty} V(j) q_{j}$ converges, then $\sum_{j=n_{0}}^{\infty} q_{j}$ converges and

$$
\left|V(n) \sum_{j=n}^{\infty} q_{j}\right| \leq(1+K) \rho_{n}, \quad n \geq n_{0}
$$

where

$$
\rho_{n}=\sup _{\ell \geq n}\left|\sum_{j=\ell}^{\infty} V(j) q_{\jmath}\right|
$$

PROOF. With

$$
p_{n}=\sum_{j=n}^{\infty} V(j) q_{\jmath}
$$

using summation by parts and the fundamental Theorem of sum calculus, we have

$$
\begin{aligned}
\sum_{j=n}^{n_{1}-1} & =\sum_{j=n}^{n_{1}-1} V^{-1}(j) V(j) q_{j} \\
& =-\left.V^{-1}(j) p_{j}\right|_{n} ^{n_{1}}+\sum_{j=n}^{n_{1}-1} \Delta\left(V^{-1}(j)\right) p_{\jmath+1} .
\end{aligned}
$$

From (2.3), (2.5) and (2 6),

$$
\left|\Delta\left(V^{-1}(j)\right) p_{\jmath+1}\right| \leq \rho_{n_{0}}\left|\Delta\left(V^{-1}(j)\right)\right|, \quad j \geq n_{0} ;
$$

and

$$
\lim _{n_{1} \rightarrow \infty} V^{-1}\left(n_{1}\right) p_{n_{1}}=0
$$

hence, because of (2.2), we can let $n_{1} \rightarrow \infty$ in (2.7) we obtain

$$
\sum_{j=n}^{\infty} q_{\jmath}=V^{-1}(n) p_{n}+\sum_{j=n}^{\infty} \Delta\left(V^{-1}(j)\right) p_{\jmath+1} .
$$

Multiplying by $V(n)$, we obtain

$$
V(n) \sum_{j=n}^{\infty} q_{\jmath}=p_{n}+V(n) \sum_{j=n}^{\infty} \Delta\left(V^{-1}(j)\right) p_{\jmath+1}
$$

Thus, by (2.1), (2.5) and (2.6)

$$
\begin{aligned}
\left|V(n) \sum_{j=n}^{\infty}\right| q_{\jmath} & \leq\left|p_{n}\right|+\sum_{j=n}^{\infty}\left|V(n) \triangle\left(V^{-1}(j)\right)\right|\left|p_{\jmath+1}\right| \\
& \leq\left|p_{n}\right|+\left|\rho_{n}\right| \cdot \sum_{j=n}^{\infty}\left|V(n) \Delta\left(V^{-1}(j)\right)\right| \\
& \leq(1+K) \rho_{n}, \quad n \geq n_{0} .
\end{aligned}
$$

DEFINITION 1. Let $\mathcal{H}\left(n_{0}\right)$ be the Banach space of sequences $h: N \rightarrow \mathbf{R}^{m}$ such that $V h$ is bounded, with norm

If $\lambda>0$, let

$$
\|h\|=\sup _{n \geq n_{0}}\left|V(n) h_{n}\right| .
$$

$$
\mathcal{H}\left(n_{0}\right)=\left\{h \in \mathcal{H}\left(n_{0}\right)\|h\| \leq \lambda\right\} .
$$


In addition, we need the following definition

DEFINITION 2. (See [7]) A vector $\xi$ is a Lipschitz point of a vector function $\psi$ if there are constants $r, c>0$ such that $\psi(x)$ is defined whenever

$$
|x-\xi| \leq r
$$

and

$$
\left|\psi\left(x^{1}\right)-\psi\left(x^{2}\right)\right| \leq c\left|x^{1}-x^{2}\right|
$$

lf $\left|x^{2}-\xi\right| \leq r ; i=1,2$.

\section{MAIN RESULTS}

The following is our main theorem

THEOREM 1. For a given vector $\xi$, suppose there are constant $\lambda>0$ and $n_{0} \in \mathbf{N}$ such that the function $f$ is defined on the set

$$
U=\left\{(n, x) \| V(n)(x-\xi) \mid \leq \lambda, n \geq n_{0}\right\},
$$

and the serles

$$
I(n ; h)=\sum_{\jmath=n}^{\infty} V(\jmath) f\left(j, \xi+h_{\jmath}\right), \quad n \geq n_{0}
$$

converges if $h \in \mathcal{H}_{\lambda}\left(n_{0}\right)$. Suppose also that

$$
\left|I\left(n ; h^{1}\right)-I\left(n ; h^{2}\right)\right| \leq \delta\left\|h^{1}-h^{2}\right\|, \quad n \geq n_{0},
$$

whenever $h^{1}, h^{2} \in \mathcal{H}_{\lambda}\left(n_{0}\right)$, where

$$
0 \leq \delta \leq 1 /(1+K)
$$

Then Eq. (1 1) has a solution $x$ which is defined for $n$ sufficiently large and satısfies

$$
\lim _{n \rightarrow \infty} V(n)\left(x_{n}-\xi\right)=0
$$

Moreover, if $y$ is any solution of Eq. (1 1 ) such that

$$
\lim _{n \rightarrow \infty} V(n)\left(y_{n}-\xi\right)=0 \text {, }
$$

then $x_{n}=y_{n}$, for $n$ sufficiently large.

PROOF. If $h \in \mathcal{H}_{\lambda}\left(n_{0}\right)$ then from (3.3)

$$
\begin{aligned}
|I(n ; h)| & \leq|I(n ; h)-I(n ; 0)|+|I(n ; 0)|, \\
& \leq \delta \lambda+|I(n ; 0)| .
\end{aligned}
$$

Now choose $n_{1} \geq n_{0}$ so that

$$
\delta \lambda+\sup _{n \geq n_{1}}|I(n ; 0)| \leq \lambda /(1+K),
$$

which is possible because of $(34)$ and the convergence of

From (3.7) and (3 8),

$$
I(n ; 0)=\sum_{j=n}^{\infty} V(j) f(j, \xi) .
$$

$$
|I(n ; h)| \leq \lambda /(1+K) \text { if } n \geq n_{1},
$$

and $h \in \mathcal{H}_{\lambda}\left(n_{0}\right)$. If $h \in \mathcal{H}_{\lambda}\left(n_{0}\right)$, define $\mathcal{Y} h$ by

$$
\mathcal{Y} h_{n}=-\sum_{j=n}^{\infty} f\left(j, \xi+h_{\jmath}\right), \quad n \geq n_{1} .
$$

From (3.2) and Lemma 1 with $q_{n}=f\left(n, \xi+h_{n}\right), \mathcal{Y} h$ is defined and satisfies the inequality

$$
\left|V(n) \mathcal{Y} h_{n}\right| \leq(1+K) \sup _{\ell \geq n}|I(\ell ; h)|, \quad n \geq n_{1}
$$


From this and (39),

$$
\left|V(n) \mathcal{Y} h_{n}\right| \leq \lambda, \quad n \geq n_{1}
$$

Therefore $\mathcal{Y} h \in \mathcal{H}_{\lambda}\left(n_{1}\right)$, that is, $\mathcal{Y}$ transforms $\mathcal{H}_{\lambda}\left(n_{1}\right)$ into itself. Now, suppose $h^{\iota} \in \mathcal{H}_{\lambda}\left(n_{1}\right)$, $(i=1,2) \quad$ Then Lema 1 with $q_{n}=f\left(n, \xi+h_{n}^{1}\right)-f\left(n, \xi+h_{n}^{2}\right)$ implies that

$$
\left|V(n)\left(\mathcal{Y} h_{n}^{1}-\mathcal{Y} h_{n}^{2}\right)\right| \leq(1+K)\left|I\left(n ; h^{1}\right)-I\left(n ; h^{2}\right)\right|, \quad n \geq n_{1}
$$

and so, from (2 8) and ( 33 ) (with $n_{0}=n_{1}$ ),

$$
\left\|\mathcal{Y} h^{1}-\mathcal{Y} h^{2}\right\| \leq \delta(1+K)\left\|h^{1}-h^{2}\right\|
$$

Hence, from (34), $\mathcal{Y}$ is a contraction mappings of $\mathcal{H}_{\lambda}\left(n_{1}\right)$ into itself, and therefore there is an $h^{0} \in \mathcal{H}_{\lambda}\left(n_{1}\right)$ such that $h^{0}=\mathcal{Y} h^{0}$, that is

$$
h_{n}^{0}=-\sum_{\jmath=n}^{\infty} f\left(j, \xi+h_{\jmath}^{0}\right), \quad n \geq n_{1} .
$$

From Lemma 1, with $q_{n}=f\left(n, \xi+h_{n}^{0}\right), \lim _{n \rightarrow \infty} V(n) h_{n}^{0}=0$. Therefore the function $x=\xi+h^{0}$ satisfies $\mathrm{Eq}$ (1.1) and (3 5) If $y$ satisfies Eq (1.1) and (3.6), then $h^{1}=y-\xi$ is in $\mathcal{H}_{\lambda}\left(n_{0}\right)$ for some $n_{2} \geq n_{1}$, and

$$
h_{n}^{1}-h_{n}^{0}=\sum_{\jmath=n}^{\infty}\left[f\left(j, \xi+h_{\jmath}^{0}\right)-f\left(j, \xi+h_{\jmath}^{1}\right)\right], \quad n \geq n_{2} .
$$

By an argument like that which led to (3 10),

$$
\left\|h^{1}-h^{0}\right\| \leq \delta(1+K)\left\|h^{1}-h^{0}\right\|
$$

which implies that $h_{n}^{1}=h_{n}^{0}$ for $n \geq n_{2}$, because of (34) This implies that $x_{n}=y_{n}$, for $n$ sufficiently large.

We now apply Theorem 1 to the system

$$
\triangle x_{n}=a(n) \psi\left(x_{n}\right)+g_{n}, \quad n \geq n_{0}
$$

THEOREM 2. Suppose $A$ is an $m \times \ell$ matrix function and $g$ is an $m$-vector function, both defined on $N$, and $\xi$ is a Lipschitz point of the $\ell$-vector function $\psi$. Suppose also that

$$
\sum_{j=n_{0}}^{\infty} V(j)\left[A(j) \psi(\xi)+g_{j}\right]
$$

converges and

$$
\sum_{j=n_{0}}^{\infty}|V(j) A(j)|\left|V^{-1}(j)\right|<\infty .
$$

Then the conclusion of Theorem I holds for Eq. (3.11).

PROOF. Let

$$
\sigma=\sup _{n \geq n_{1}}\left|V^{-1}(n)\right|
$$

which is finite because of (2.3). Let $\delta$ be any number that satisfies (3.4), let $c$ be as in (2.9), and choose $n_{2}$ so that

$$
c \sum_{j=n_{2}}^{\infty}|V(j) A(j)|\left|V^{-1}(j)\right| \leq \delta,
$$

which is possible because of (3.13). Henceforth, let $n \geq n_{2}$. Finally, let

$$
\lambda=r / \sigma
$$

with $r$ as in (2.9). We will show that $\lambda$ satisfies the requirement of Theorem 1 , for

$$
f(n, x)=A(n) \psi(x)+g_{n}
$$

We must first show that $f(n, x)$ is defined on $U$, for $U$ given as in (12). If $(n, x) \in U$, then 


$$
|x-\xi| \leq\left|V^{1}(n)\right||V(n)(x-\xi)| \leq \sigma \lambda=r,
$$

because of (3 14) and (3 16) Since $\psi$ is defined for all $x$ satisfying (29), while $A$ and $g$ are defined on $N$, it follows that $f(n, x)$ is defined on $U$, and $f\left(n, \xi+h_{n}\right)$ is defined for $n \geq n_{1} \geq n_{0}$ if $h \in \mathcal{H}_{\lambda}\left(n_{0}\right)$ Moreover, if $h^{1}, h^{2} \in \mathcal{H}_{\lambda}\left(n_{0}\right)$, then

$$
\begin{aligned}
\left|\psi\left(\xi+h_{n}^{1}\right)-\psi\left(\xi+h_{n}^{2}\right)\right| & \leq c\left|h_{n}^{1}-h_{n}^{2}\right| \\
& \leq c\left|V^{-1}(n)\right|\left|V(n)\left(h_{n}^{1}-h_{n}^{2}\right)\right| \\
& \leq c\left|V^{-1}(n)\right|\left\|h^{1}-h^{2}\right\|, \quad n \geq n_{2} ;
\end{aligned}
$$

by (2 8$)$ and (2 1) This, ( $\left.\begin{array}{ll}3 & 15\end{array}\right)$ and ( 318$)$ imply that

$$
\left|\sum_{\jmath=n}^{\infty} V(j) A\left[\psi\left(\xi+h_{\jmath}^{1}\right)-\psi\left(\xi+h_{\jmath}^{2}\right)\right]\right| \leq \delta\left\|h^{1}-h^{2}\right\|, \quad n \geq n_{2}
$$

With $f$ as in (3 17), the functional $I$ in $(32)$ becomes $I(n ; h)=\sum_{j=n}^{\infty} V(j)\left[A(j) \psi\left(\xi+h_{\jmath}\right)+g_{\jmath}\right]$ From the convergence of $(312), I(n ; 0)$ exists This and the convergence of the series in (3 19) with $h^{1}=h$ and $h^{2}=0$ imply that $I(n ; h)$ exists for all $h \in \mathcal{H}_{\lambda}\left(n_{0}\right)$, if $n \geq n_{2} \geq n_{1} \quad$ Knowing this, we can conclude from (3 19) that (3 3) holds whenever $h^{1}, h^{2} \in \mathcal{H}_{\lambda}\left(n_{0}\right)$. This completes the proof of Theorem 2

Stronger results are available for a linear system

$$
\triangle x_{n}=A(n) x_{n}+g_{n}, \quad n \geq n_{0} .
$$

THEOREM 3. Suppose that for an $m \times m$ matrix function $A$ and an $m$-vector function $g$ defined on $N$,

and

$$
\sum_{j=n_{0}}^{\infty}\left|V(j) A(j) V^{-1}(J)\right|<\infty
$$

$$
\sum_{j=n_{0}}^{\infty} V(j)\left[A(j) \xi+g_{j}\right]
$$

converges for a given constant vector $\xi$. Then Eq. (3.20) has a unique solutıon $x$ which satisfies (3.5)

PROOF. Taking $\psi(x)=x$, the proof is similar to that of Theorem 2 for a given constant vector $\xi$

The next theorem follows from this and elementary properties of linear difference systems.

THEOREM 4. Suppose $A$ and $g$ are defined on $N,(3.21)$ holds and $\sum_{j=n_{0}}^{\infty} V(j) A(j)$ and $\sum_{j=n_{0}}^{\infty} V(j) g_{j}$ converge. Then Eq. (3.20) has a unique solution which satisfies (3.5) for any given constant vector $\xi$; and every solution of Eq. (3.22) satisfies (3.5) for some $\xi$.

PROOF. Any constant vector $\xi$ is a Lipschitz point of $\psi(x)=x$. Moreover, if $\sum_{j=n_{0}}^{\infty} V(j) A(j)$ converges, then $\sum_{j=n_{0}}^{\infty} V(j) A(j) \xi$ converges, too. From this, the series $\sum_{j=n_{0}}^{\infty} V(j)\left[A(j) \xi+g_{j}\right]$ converges for any constant vector $\xi$. Therefore, for every constant vector $\xi$, Theorem 3 ensures that there is a unique solution of Eq. $(322)$ satisfying (3.5). The second statement of Theorem 4 follows the uniqueness given by (3.6) in Theorem 1 and properties of linear difference systems.

EXAMPLE 1. The difference system

$$
\left[\begin{array}{c}
\triangle x_{1} \\
\Delta x_{2}
\end{array}\right]=\frac{\cos (n)}{n^{4}\left(x_{1}-x_{2}\right)^{2}}\left[\begin{array}{cc}
3 & 7-n \\
n^{2} & n-2
\end{array}\right]\left[\begin{array}{c}
x_{1}^{2} \\
x_{1} x_{2} \\
x_{2}
\end{array}\right]+\frac{\cos (n)}{n^{5}}\left[\begin{array}{c}
10 \\
4-n
\end{array}\right],
$$

has the form (3.11), for $n \geq 1$.

If $V(n)=\operatorname{diag}\left(n^{\mu}, n^{\mu-1}\right)$ with $\mu \geq 1$, then (3.13) holds. If $\xi_{1} \neq \xi_{2}$, then straightforward calculations show that $\xi=\left(\xi_{1}, \xi_{2}\right)$ is a Lipschitz point of $\psi$ in (3.23), and (312) converges if $\mu<4$. Therefore Theorem 2 implies that Eq (3 23) has a solution $x=\left(x_{1}, x_{2}\right)$ such that 


$$
\begin{aligned}
& x_{1}(n)=\xi_{1}+o\left(n^{-\mu}\right), \\
& x_{2}(n)=\xi_{2}+o\left(n^{-\mu+1}\right),
\end{aligned}
$$

as $n \rightarrow \infty$ for all $\mu<4$, provided $\xi_{1} \neq \xi_{2}$

EXAMPLE 2. We now exhibit a system $\triangle x=A(n) x$ whose solutions all tend to constant vectors, even though $\Sigma^{\infty}|A(n)|=\infty$ To this end, we observe that if $\mu<r, \sum_{j=1}^{\infty} \jmath^{-r} e^{\mu \jmath} \cos \left(e^{\jmath}\right)$ converges for all $r>0$

Now consider the system

$$
\left[\begin{array}{c}
\Delta x_{1} \\
\Delta x_{2}
\end{array}\right]=\cos \left(e^{n}\right)\left[\begin{array}{cc}
a n^{-3 / 2} & b n^{-1 / 2} \\
c n^{-5 / 2} & d n^{-3 / 2}
\end{array}\right]\left[\begin{array}{l}
x_{1} \\
x_{2}
\end{array}\right],
$$

where $a, b, c, d$ are constants and $b \neq 0$, so that $\sum_{n=1}^{\infty}|A(n)|=\infty$, and let $V(n)=\operatorname{diag}\left(e^{\mu n}, n e^{\mu n}\right)$, with $0 \leq \mu<\frac{1}{2} \quad$ Here $\sum_{n=1}^{\infty} V(n) A(n)$ converges and (3.21) holds, hence Theorem 4 implies that if $\xi_{1}$ and $\xi_{2}$ are arbitrary, then $(324)$ has a solution such that

as $n \rightarrow \infty$

$$
\begin{aligned}
& x_{1}(n)=\xi_{1}+o\left(e^{-\mu n}\right), \\
& x_{2}(n)=\xi_{2}+o\left(n^{-1} e^{-\mu n}\right),
\end{aligned}
$$

ACKNOWLEDGMENT. We thank the referees for their useful observations This work has been supported by Fondecyt 92-0148 and Fondecyt 1930839

\section{REFERENCES}

[1] BRAUER, F, Asymptotic equivalence and asymptotic behavior of linear systems, Michigan Math. J. 9 (1962), 33-43

[2] DROZDOWICZ, A and POPENDA, J , Asymptotic behavior of the solutions of the second order difference equation, Proc. Amer. Math. Soc. 99 (1987), 135-140

[3] CASTILLO, S and PINTO, M, Asymptotic behavior of the solutions of second order difference equation, Proceedings of First Internatıonal Conference on Difference Eqs. Texas (1994), Gordon Beach, to appear

[4] ONUCHIC, N , Nonlinear perturbations of a linear system of ordinary differential equations, Michigan Math. J. 11 (1964), 237-242.

[5] SZMANDA, B, Nonoscillation, oscillation and growth of solutions of nonlinear difference equations of second order, J. Math. Anal. Appl. 109 No 1 (1985), 22-30

[6] TRENCH, WF, On the asymptotic behavior of solutions of second order linear differential equations, Proc. Amer. Math. Soc. 14 (1963), 12-14.

[7] TRENCH, W.F., Systems of differential equations subject to mild integral conditions, Proc. Amer. Math. Soc. 87 No 2 (1983), 263-270

[8] HANDERSON, D and PETERSON, A., A classification of the solutions of a difference equation according to their behavior at infinity, J. Math. Anal. Appl. 136 No. 1 (1988), 249-266.

[9] SZAFRANSKI, Z and SZMANDA, B., Oscillatory properties of solutions of some difference systems, Radovi Math. 6 (1990), 205-214

[10] MEDINA, R. and PINTO, M, Asymptotic behavior of solutions of second order nonlinear difference equations, J. Nonlinear Anal., T.M.A. 19 No. 2 (1992), 187-195

[11] MEDINA, R. and PINTO, M., Asymptotic representation of solutions of linear second order difference equations, J. Math. Anal. Appl. 165 (1992), No 2, 505-516

[12] MEDINA, R and PINTO, M, Asymptotic equilibrium of nonlinear difference systems, Submitted.

[13] MEDINA, R. and PINTO, M, Bounded and convergence solutions of a difference equation, Proceedings of First International Conference on Difference Equations, Ed. S Elaydi (1994)

[14] MEDINA, R and PINTO, M., Convergence of solutions of quasilinear difference equations, Submitted

[15] AGARWAL, R.P., Difference Equatıons and Inequalities, Marcel Decker, New York (1992)

[16] PINTO, M, Discrete dichotomies, Comput. Math. Appl. 28 (1994), 259-270

[17] PINTO, M., Asymptotic equivalence of difference systems, J. Difference Eqs. and Appl. (1995)

[18] NAULIN, R. and PINTO, M, Stability dichotomies for linear difference equations, J. Difference Eqs. and Appl. (to appear) 


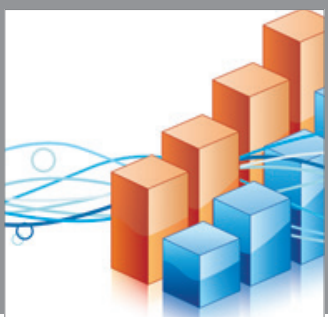

Advances in

Operations Research

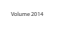

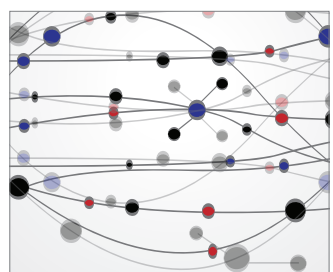

\section{The Scientific} World Journal
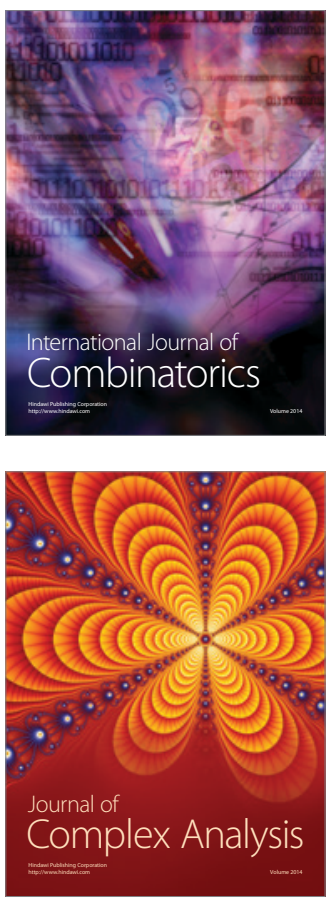

International Journal of

Mathematics and

Mathematical

Sciences
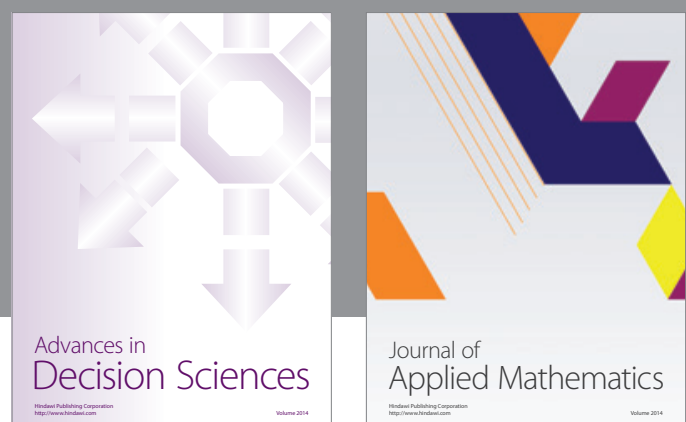

Journal of

Applied Mathematics
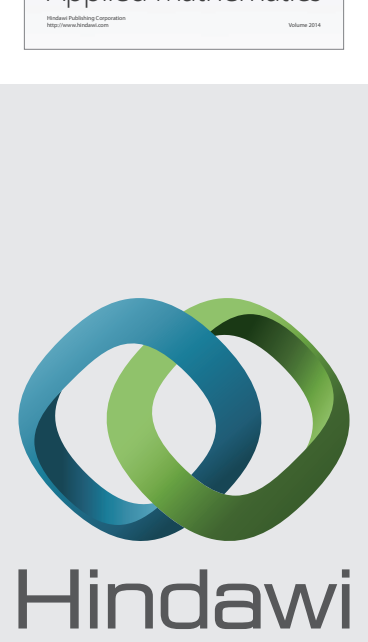

Submit your manuscripts at http://www.hindawi.com
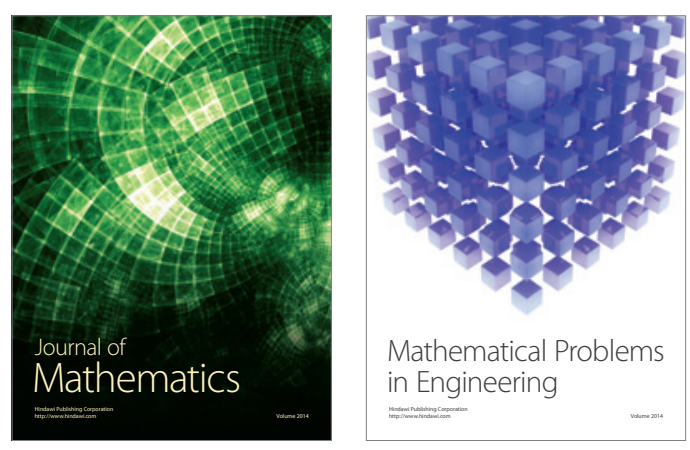

Mathematical Problems in Engineering
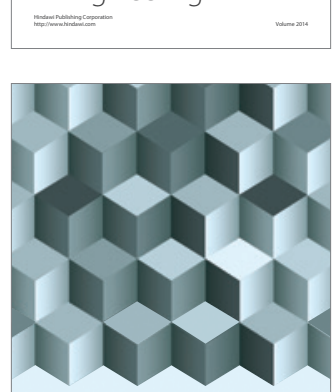

Journal of

Function Spaces
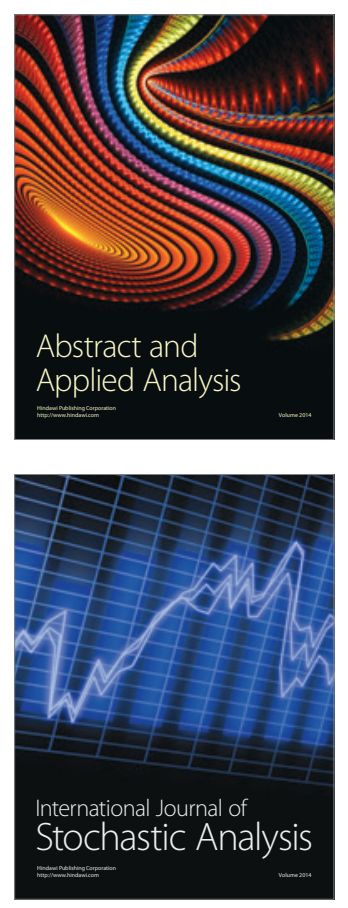

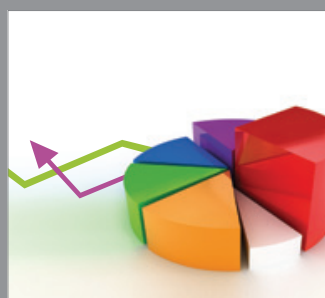

ournal of

Probability and Statistics

Promensencen
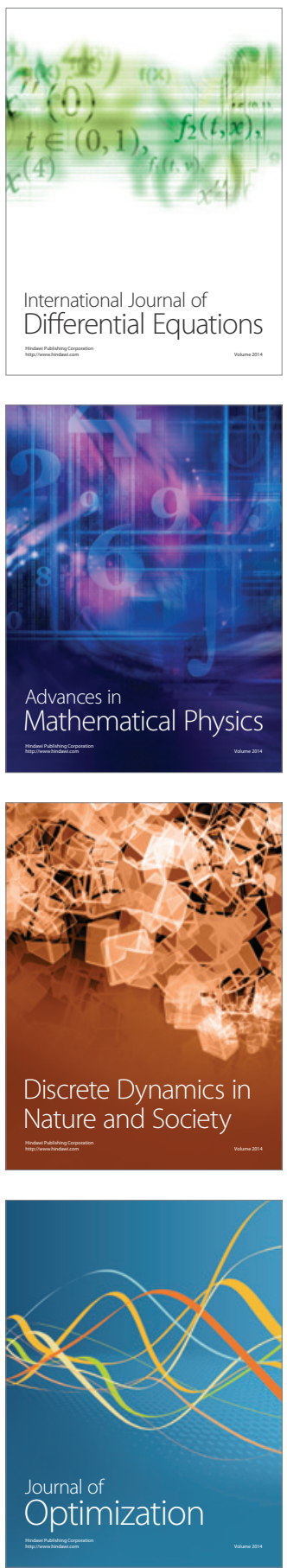